\title{
Sample preparation technologies for serial synchrotron crystallography and single-particle cryo-EM \\ Robert Thorne $^{1}$ \\ ${ }^{1}$ MiTeGen, LLC \\ ret6@cornell.edu
}

In the last decade, a wide array of sample preparation and delivery technologies have been demonstrated for XFELand synchrotron-based serial crystallography. Drawing upon this work, we have developed an integrated system that addresses key issues in serial crystallography in a robust way while maintaining flexibility required to address diverse real-world crystal handling challenges [1]. The key elements of this system are: (1) sample supports incorporating microfabricated thin films that are fully compatible with existing infrastructure for high-throughput cryo-crystallography, allow efficient removal of excess surrounding solvent and positioning of microcrystals at particular locations, generate ultra-low-background scatter while allowing easy optical imaging, and that allow both room-temperature data collection and rapid cooling for cryogenic data collection; (2) a sample loading station that allows easy dispensing and subsequent removal of liquid (e.g. ligand- and/or cryoprotectant-containing solutions, buffer to facilitate dispersing or positioning crystals) from the sample supports via precisely controllable timevarying suction; and (3) a humid "gloveless" glovebox for crystallization tray manipulations, crystal soaking, and sample support loading and sealing that, unlike commercial humidity chambers, can generate and maintain the near saturating humidities ( $>95 \%$ r.h.) required to maintain microcrystals at their as-grown hydration and maximize crystal isomorphism while maximizing allowable working times. This system's ease of use, flexibility, and optimized performance make it attractive not just for serial microcrystal crystallography but also for routine singleand few-crystal data collection.

Also in the last decade, major advances in instrumentation and data analysis have dramatically improved the achievable resolution in single-particle cryo-EM and revolutionized its application in structural biology. As in crystallography, sample preparation is the key bottleneck in cryo-EM. Motivated by fundamental physical insights, we have demonstrated novel approaches to aspects of sample preparation that promise to simplify workflows and improve data quality [2].

[1] G. Illava et al. Acta Cryst. D77, 628-644 (2021).

[2] T. Engstrom et al. (submitted). 